S4 Table Association of monthly minimum wage with obesity in adult women using pooled data with interaction terms

\section{All countries}

Odds ratios (CI95)

$0.9985^{\star \star *}$

$0.9981,0.9989$

Low-income countries

Middle-income countries

Predicted prevalence difference

$-.00015^{\star \star *} \quad-.0002,-.0001$

$1.0043^{\star * *}$

$1.0029,1.0057$

$0.9979 * * *$

$0.9976,0.9982$

$-.00032$

$-.00036,-.00027$

Odds ratios $(95 \% \mathrm{Cl})$ obtained by two-level random intercept model. Development-specific estimates with interaction term between minimum wage (continuous) and income group (binary). Sample restricted to adult women (24-49 y), $n=162,446$. Level 2 residual variance was 0.6538 (SE .233). Variance Partition Coefficient (VPC) $=0.166$. Post-estimation of predicted probability of being overweight with 1-unit change in minimum wage obtained using average marginal effects with respect to minimum wage over country income group. ${ }^{* * *} p<0.001,{ }^{* *} p<0.01,{ }^{*} p<0.05$. 